\title{
ZEROS OF GAUSSIAN ANALYTIC FUNCTIONS
}

\author{
M. SODIN
}

\section{Introduction}

Throughout this note we shall use the following notation. Let $G \subseteq \mathbb{C}^{1}$ be a plane domain and $\left\{\psi_{j}(z)\right\}_{j=1}^{N}$ be a system of $N \leq \infty$ analytic functions in $G$. By $\Psi(z)$ we denote the holomorphic curve in the euclidean space $\mathbb{C}^{N}$ with coordinates $\psi_{j}(z)$. If $N=\infty$, we assume that

$$
\|\Psi(z)\|=\sum_{j}\left|\psi_{j}(z)\right|^{2}<\infty, \quad z \in G,
$$

where the series on the RHS converges locally uniformly in $G$.

Let $\omega_{j}$ be independent, complex-valued, gaussian random variables such that

$$
\mathcal{E}\left\{\omega_{j}\right\}=0, \quad \text { and } \quad \mathcal{E}\left\{\left|\omega_{\mathrm{j}}\right|^{2}\right\}=1 .
$$

We identify the probability space with $\mathbb{C}^{N}$ equipped with the gaussian product measure $d \nu$.

A gaussian analytic function $\psi(z, \omega)$ is defined as

$$
\psi(z, \omega)=\sum_{j} \omega_{j} \psi_{j}(z)
$$

(cf. [15]). If $N=\infty$, then according to a theorem by Khintchin and Kolmogorov (cf. [14, Chapter 3, Theorem 2]), the series converges locally uniformly in $G$ and almost surely in $\omega$, and hence defines an analytic function in $G$.

Let $n_{\omega}$ be a counting measure of zeros (according to their multiplicities) of the function $\psi(z, \omega)$. Here we shall be concerned with three general results on the random measure $n_{\omega}$. The first one is a formula for the average $\mathcal{E}\left\{n_{\omega}\right\}$ which is due to Edelman and Kostlan (cf. [7, Theorem 8.1]). The second, close to Calabi's Rigidity Theorem [4], loosely speaking says that the average measure $\mathcal{E}\left\{n_{\omega}\right\}$ "almost determines" the analytic functions $\psi_{j}(z)$. The third result, which is due to Offord [22] (cf. [23] and [24]), is an exponential decrease of "tail probabilities" of an analytic function having an excess or deficiency of zeros in a given region. An important feature of these three results is that they do not need any assumptions about analytic functions $\psi_{j}(z)$ and "dimension" $N$. We shall not touch on the more delicate statistics of the local correlation functions, which was

Received May 31, 1999.

Partially supported by grant number 96-00030 of the United States - Israel Binational Science Foundation, and by grant number 93/97.1 of the Israel Science Foundation of the Israel Academy of Sciences and Humanities. 
recently of some interest in mathematical physics (see references at the end of this note).

By $C$ and $c$ we denote various positive numerical constants which may vary from line to line.

\section{Edelman-Kostlan formula for the average number of zeros}

Theorem 1. In the assumptions formulated above,

$$
\mathcal{E}\left\{n_{\omega}\right\}=\frac{1}{2 \pi} \Delta \log \|\Psi\| d m(z),
$$

where $\Delta$ is a distributional Laplacian and $d m(z)$ is the plane Lebesgue measure; i.e., the average $\mathcal{E}\left\{n_{\omega}\right\}$ coincides with the Riesz measure of the subharmonic function $\log \|\Psi(z)\|$.

For $N<\infty$, Edelman and Kostlan outlined the proof. In a special case, the proof appears in [26, Lemma 3.1].

It is curious to note that since

$$
\mathcal{E}\left\{|\psi(z, \omega)|^{2}\right\}=\sum_{i, j} \mathcal{E}\left\{\omega_{i} \overline{\omega_{j}}\right\} \psi_{i}(z) \overline{\psi_{j}(z)}=\|\Psi(z)\|^{2},
$$

equation (3) can be rewritten in the form

$$
\mathcal{E}\left\{\frac{1}{2 \pi} \Delta \log |\psi(z, \omega)|^{2}\right\}=\frac{1}{2 \pi} \Delta \log \mathcal{E}\left\{|\psi(z, \omega)|^{2}\right\} .
$$

Proof of Theorem 1. Let $\phi$ be a test function with a compact support in $G$. Then, according to Green's formula,

$$
\int_{G} \phi(z) d n_{\omega}(z)=\frac{1}{2 \pi} \int(\Delta \phi)(z) \log |\psi(z, \omega)| d m(z) .
$$

First, we assume that $N<\infty$. In this case we repeat verbatim the argument from [26, Lemma 3.1]. We integrate the both sides of formula (5) against the gaussian measure $d \nu$ in $\mathbb{C}^{N}$. Changing the order of integration on the RHS and using the notation $(a, b)=\sum_{j} a_{j} b_{j}$ for the scalar product in $\mathbb{C}^{N}$ without complex conjugation, and $\hat{a}=a /\|a\|$, we obtain

$$
\begin{aligned}
\int_{G} \phi(z) \mathcal{E}\left\{d n_{\omega}(z)\right\}= & \frac{1}{2 \pi} \int_{G}(\Delta \phi)(z) d m(z) \int_{\mathbb{C}^{N}} \log |(\Psi(z), \omega)| d \nu(\omega) \\
= & \frac{1}{2 \pi} \int_{G}(\Delta \phi)(z) d m(z)\{\log || \Psi(z) \| \\
& \left.+\int_{\mathbb{C}^{N}} \log || \omega \| d \nu(\omega)+\int_{\mathbb{C}^{N}} \log |(\hat{\Psi}(z), \hat{\omega})| d \nu(\omega)\right\} .
\end{aligned}
$$

The second integral in the braces does not depend on $z$. The third integral in the braces also does not depend on $z$ because of the rotational invariance of the gaussian measure $d \nu$. Hence, by the Gauss formula, after integration against 
$(\Delta \phi)(z)$, where $\phi$ is compactly supported in $G$, these two terms vanish, and we obtain

$$
\int_{G} \phi(z) \mathcal{E}\left\{d n_{\omega}(z)\right\}=\frac{1}{2 \pi} \int_{G}(\Delta \phi)(z) \log \|\Psi(z)\| d m(z),
$$

which is equivalent to (3).

Now, consider the case $N=\infty$. Clearly, we no longer have rotational invariance of $d \nu$. Instead, we shall use an approximation argument together with the following fact which will be proved in Section 4: for each $z \in G$, and each $\lambda>0$,

$$
\operatorname{Pr}(\{\omega:|\log | \psi(z, \omega)|-\log \|\Psi(z)\||>\lambda\}) \leq 3 e^{-\lambda} .
$$

This yields that for every compact subset $K$ of $G$

$$
\int_{\mathbb{C}^{\infty}} d \nu(\omega) \int_{K} \log ^{2}|\psi(z, \omega)| d m(z) \leq C m(K)+2 \int_{K} \log ^{2}\|\Psi(z)\| d m(z) .
$$

Let $k$ be a positive integer. Denote by $\Psi_{k}(z)$ a holomorphic curve in $\mathbb{C}^{k}$ which consists of the first $k$ components of the curve $\Psi(z)$, and set $\psi_{k}(z, \omega)=$ $\left(\Psi_{k}(z), \omega\right)$. For a.a. $\omega \in \mathbb{C}^{\infty}$, the sequence of analytic functions $z \mapsto \psi_{k}(z, \omega)$ converges to $\psi(z, \omega)$ locally uniformly in $G$, and therefore

$$
\lim _{k \rightarrow \infty} \int_{K}|\log | \psi(z, \omega)|-\log | \psi_{k}(z, \omega)|| d m(z)=0 .
$$

On the other hand, applying estimate (7) to the functions $\psi_{k}(z, \omega)$, and using the locally uniform convergence of $\log \left\|\Psi_{k}(z)\right\|$ to $\log \|\Psi(z)\|$, we get:

$$
\int_{\mathbb{C}^{\infty}} d \nu(\omega) \int_{K} \log ^{2}\left|\psi_{k}(z, \omega)\right| d m(z) \leq C m(K)+3 \int_{K} \log ^{2}\|\Psi(z)\| d m(z),
$$

if $k$ is sufficiently large.

Put

$$
h_{k}(\omega)=\int_{K}|\log | \psi_{k}(z, \omega)|-\log | \psi(z, \omega)|| d m(z) .
$$

Then, by (8),

$$
\lim _{k \rightarrow \infty} h_{k}(\omega)=0, \quad \nu \text { - a.e }
$$

and by (7) and (9)

$$
\int_{\mathbb{C}^{\infty}} h_{k}^{2}(\omega) d \nu(\omega) \leq M(K),
$$

where the constant $M(K)$ does not depend on $k$.

Relations (10) and (11) yield that

$$
\lim _{k \rightarrow \infty} \int_{\mathbb{C}^{\infty}} h_{k}(\omega) d \nu(\omega)=0,
$$

or

$$
\lim _{k \rightarrow \infty} \int_{K} d m(z) \int_{\mathbb{C}^{\infty}}|\log | \psi_{k}(z, \omega)|-\log | \psi(z, \omega)|| d \nu(\omega)=0 .
$$


Since $K$ is an arbitrary compact subset of $G$, this yields that the sequence of subharmonic functions

$$
z \mapsto \int_{\mathbb{C}^{\infty}} \log \left|\psi_{k}(z, \omega)\right| d \nu(\omega), \quad k=1,2, \ldots, \quad z \in G,
$$

converges in $L_{\text {loc }}^{1}(G)$ to the subharmonic function

$$
z \mapsto \int_{\mathbb{C}^{\infty}} \log |\psi(z, \omega)| d \nu(\omega), \quad z \in G .
$$

By continuity of the Laplacian in $L_{\text {loc }}^{1}(G)$, this yields weak convergence of the corresponding Riesz measures. That is, for $k \rightarrow \infty$, the sequence $\mathcal{E}\left\{n_{\omega, k}\right\}$ weakly converges to $\mathcal{E}\left\{n_{\omega}\right\}$, where $n_{\omega, k}$ is the counting measure of zeros of $\psi_{k}(z, \omega)$. Besides, the Riesz measures of $\log \left\|\Psi_{k}(z)\right\|$ also converge weakly to the Riesz measures of $\log \|\Psi(z)\|$. By the first part of the proof, $\mathcal{E}\left\{n_{\omega, k}\right\}$ coincides with the Riesz measure of $\log \left\|\Psi_{k}(z)\right\|$. This completes the proof.

\section{Calabi's Rigidity}

In this section, we assume that the functions $\psi_{j}$ are linearly independent, that is, if

$$
\sum_{j} c_{j} \psi_{j}(z) \equiv 0, \quad \sum_{j}\left|c_{j}\right|^{2}<\infty
$$

then all $c_{j}$ must vanish.

We are interested in the following question:

- What we are allowed to do with the functions $\psi_{j}(z)$ witnout changing the average measure $\mathcal{E}\left\{n_{\omega}\right\}$ ?

Surely, we are allowed to multiply all functions $\psi_{j}$ by the same analytic function $g$ without zeros in $G$. We can also apply a constant unitary transformation $U$ to the vector-function $\Psi(z)$ since this does not change the distribution of the function $\psi(z, \omega)$. Amazingly enough, the measure $\mathcal{E}\left\{n_{\omega}\right\}$ determines the functions $\psi_{j}(z)$ (and the dimension $N$ ) up to these operations.

Theorem 2. Let $\Psi_{1}(z)$ and $\Psi_{2}(z)$ be analytic vector functions in $G$ with linearly independent components and with the same average measure $\mathcal{E}\left\{n_{\omega}\right\}$ of zeros of $(\Psi(z), \omega)$. Then, the dimension $N$ of the vectors $\Psi_{1}$ and $\Psi_{2}$ is the same, and there exists a scalar analytic function $g(z)$ without zeros in $G$, and a unitary transformation $U$ in $\mathbf{C}^{N}$ such that

$$
\Psi_{2}(z)=g(z) U \Psi_{1}(z) .
$$

Proof. Having a vector-function $\Psi(z)$ with linearly independent analytic components $\psi_{j}(z)$, define the Hilbert space $\mathcal{H}$ of analytic functions in $G$ with elements

$$
f(z)=\sum_{j} c_{j} \psi_{j}(z), \quad\|f\|_{\mathcal{H}}=\sum_{j}\left|c_{j}\right|^{2} .
$$


This is a kernel space with the reproducing kernel

$$
K(z, w)=\sum_{j} \psi_{j}(z) \overline{\psi_{j}(w)}, \quad f(w)=\langle f, K(., w)\rangle_{\mathcal{H}} .
$$

The function $K(z, w)$ is analytic in $z$, anti-analytic in $w$ and is hermitianpositive. According to Theorem 1, the measure $\mu=\mathcal{E}\left\{n_{\omega}\right\}$ coincides with the Riesz measure of the subharmonic function $\log \sqrt{K(z, z)}$.

Now, assume that we have two Hilbert spaces $\mathcal{H}_{1}$ and $\mathcal{H}_{2}$ of analytic functions in $G$ with kernels $K_{1}$ and $K_{2}$ such that $\log \sqrt{K_{1}(z, z)}$ and $\log \sqrt{K_{2}(z, z)}$ have the same Riesz measure, that is, the function $\log K_{1}(z, z)-\log K_{2}(z, z)$ is harmonic in $G$. Then

$$
K_{2}(z, z)=e^{2 u(z)} K_{1}(z, z),
$$

where $u(z)$ is harmonic in $G$.

Observe that the diagonal $\{(\lambda, \lambda): \lambda \in G\}$ is a set of uniqueness for functions $K(z, w)$ analytic in $z \in G$ and anti-analytic in $w \in G$. Indeed, for a moment set $\widehat{K}(\lambda)=K(\lambda, \lambda)$. Then, for $m, n \in \mathbf{Z}_{+}$,

$$
\left.\frac{\partial^{m+n} K}{\partial z^{m} \partial \bar{w}^{n}}\right|_{(\lambda, \lambda)}=\frac{\partial^{m+n} \widehat{K}(\lambda)}{\partial \lambda^{m} \partial \bar{\lambda}^{n}} .
$$

This follows from the formal rules of taking $\partial$ and $\bar{\partial}$ derivatives. (It is enough to check this for monomials of the form $z^{k} \bar{w}^{l}, k, l \in \mathbf{Z}_{+}$.) Therefore, knowing $\widehat{K}(\lambda)$, we may use the Taylor formula and hence extend the kernel $K$ from the diagonal to its neighbourhood.

This observation shows that

$$
K_{2}(z, w)=g(z) \overline{g(w)} K_{1}(z, w),
$$

where $g(z)$ is an analytic function in $G$ without zeros, and $\log |g|=u$. Indeed, the RHSs of (13) and (14) coincide on the diagonal. Since the both kernels $K_{1}$ and $K_{2}$ are single-valued functions in $z$ and $w$, the function $g$ is also single-valued.

Having at hands the kernel $K$, one recovers the space $\mathcal{H}$ as the closure of finite linear combinations

$$
\zeta(z)=\sum_{i} \zeta_{i} K\left(z, w_{i}\right), \quad\left\{\zeta_{i}\right\} \subset \mathbb{C}, \quad\left\{w_{i}\right\} \subset G,
$$

in the norm

$$
\|\zeta\|_{\mathcal{H}}^{2}=\sum_{i, j} \zeta_{i} \overline{\zeta_{j}} K\left(w_{i}, w_{j}\right)
$$

Then equation (14) yields that

$$
\mathcal{H}_{2}=\left\{f=g h: h \in \mathcal{H}_{1}\right\}, \quad\left\langle f_{1}, f_{2}\right\rangle_{\mathcal{H}_{2}}=\left\langle h_{1}, h_{2}\right\rangle_{\mathcal{H}_{1}} .
$$

By construction, the components $\left\{\psi_{1, j}\right\}$ of $\Psi_{1}$ form an orthonormal basis in $\mathcal{H}_{1}$, while the components of $\Psi_{2}$ form an orthonormal basis in $\mathcal{H}_{2}$. Therefore, relations (15) are equivalent to (12). This completes the proof. 
An equivalent formulation can be made in the framework of differential geometry. As above, consider a holomorphic curve $\Psi(z)$ in $\mathbb{C}^{N}$ with components $\psi_{j}(z)$. The curve gives a holomorphic embedding of $G$ equipped with a Riemannian metric into euclidean $N$-dimensional space. The argument given above shows that knowing the norm of the curve

$$
\|\Psi(z)\|^{2}=\sum_{j}\left|\psi_{j}(z)\right|^{2}
$$

in a subdomain $G^{\prime} \subseteq G$, we may recover the dimension $N$ and the whole curve $\Psi(z)$ up to a constant unitary transformation of the space $\mathbb{C}^{N}$. This is a special case of Calabi's rigidity theorem [4] (cf. Problem 207 and reference to its solution in Part IV of the revised edition of the Pólya and Szegö problem book [25, pp. 34,211]).

The Riesz measure $\mu_{K}$ of the subharmonic function $\log \sqrt{K(z, z)}$ is an important invariant of the kernel Hilbert space $\mathcal{H}$ of analytic functions [6]. In this setting, a fact equivalent to Theorem 2 was also found by Nikolskii [20, Theorem 2.4 and Lemma 2.5]).

Let $K(z, w)$ be an arbitrary hermitian-positive kernel, analytic in $z \in G$, and anti-analytic in $w \in G$. As above, let $\mu_{K}$ be the Riesz measure of $\log \sqrt{K(z, z)}$. According to Theorem 2, the class of such measures possesses a strong uniqueness property: if $\mu_{K_{1}}=\mu_{K_{2}}$ in a small disk $D$ in $G$, then $\mu_{K_{1}}-\mu_{K_{2}}$ is a divisor of a meromorphic function in $G$, and the functions $K_{1}$ and $K_{2}$ are related to each other as in (14) with a meromorphic function $g$ without zeros and poles in $D$. It would be interesting to find a quantative version of this statement when the difference $\mu_{K_{1}}-\mu_{K_{2}}$ is small in $D$.

In [5], Calabi completely decribed the class of all measures $\mu_{K}$, see also Lawson's paper [16]. It seems, this description deserves a better understanding.

\section{Offord's estimate for large deviations}

Theorem 3. Let $\mu$ be the Riesz measure of the subharmonic function $\log \|\Psi(z)\|$, and let $\phi \in C_{0}^{\infty}(G)$ be an arbitrary test function with compact support in $G$. Then, for every $\lambda>0$,

$$
\operatorname{Pr}\left(\left\{\omega:\left|\int_{G} \phi\left(d n_{\omega}-\mathcal{E}\left\{d n_{\omega}\right\}\right)\right| \geq \lambda\right\}\right) \leq 3 e^{-2 \pi \lambda /\|\Delta \phi\|_{L^{1}}}
$$

We start with a lemma which is a key ingredient in Offord's approach.

Lemma Let $\mathcal{Z}(\omega)$ be a complex gaussian random variable with zero average and variance $\sigma^{2}$. Then, for every measurable subset $E$,

$$
\left|\int_{E} \log \right| \mathcal{Z}(\omega)|d \nu(\omega)-\nu(E) \log \sigma| \leq \nu(E)\left[\log \frac{1}{\nu(E)}+\frac{1}{4}\right] .
$$


Proof of Lemma. WLOG, we may assume that $\sigma=1$, otherwise we replace $\mathcal{Z}$ by $\mathcal{Z} / \sigma$. The upper bound follows at once from Jensen's inequality:

$$
\begin{aligned}
\int_{E} \log |\mathcal{Z}| d \nu & =\frac{\nu(E)}{2} \frac{1}{\nu(E)} \int_{E} \log |\mathcal{Z}|^{2} d \nu \\
& \leq \frac{\nu(E)}{2} \log \left(\frac{1}{\nu(E)} \int_{E}|\mathcal{Z}|^{2} d \nu\right) \leq \frac{\nu(E)}{2} \log \frac{1}{\nu(E)}
\end{aligned}
$$

Now, let us prove the lower bound in (17). We have

$$
\begin{aligned}
\int_{E} \log |\mathcal{Z}| d \nu & \geq-\int_{E} \log ^{-}|\mathcal{Z}| d \nu \\
& =-\int_{E,|\mathcal{Z}| \leq \nu(E)} \log ^{-}|\mathcal{Z}| d \nu-\int_{E,|\mathcal{Z}|>\nu(E)} \log ^{-}|\mathcal{Z}| d \nu \\
& \geq-\int_{0}^{\nu(E)} \frac{\operatorname{Pr}(|\mathcal{Z}| \leq s)}{s} d s-\nu(E) \log \frac{1}{\nu(E)}
\end{aligned}
$$

Since $\mathcal{Z}$ is gaussian with variance one, we can easily estimate the first integral in the RHS:

$$
\begin{aligned}
\int_{0}^{\nu(E)} \frac{\operatorname{Pr}(|\mathcal{Z}| \leq s)}{s} d s & =\int_{0}^{\nu(E)} \frac{1-e^{-s^{2} / 2}}{s} d s \\
& \leq \frac{1}{2} \int_{0}^{\nu(E)} s d s=\frac{\nu^{2}(E)}{4} \leq \frac{\nu(E)}{4}
\end{aligned}
$$

This completes the proof.

Now we turn to the

Proof of Theorem 3. Fix $\lambda>0$, and define two sets:

$$
A_{+}=\left\{\omega: \int_{G} \phi\left(d n_{\omega}-d \mu\right) \geq \lambda\right\}
$$

and

$$
A_{-}=\left\{\omega: \int_{G} \phi\left(d n_{\omega}-d \mu\right) \leq-\lambda\right\} .
$$

Using the lemma, we can easily estimate $\nu\left(A_{ \pm}\right)$from the above. First, consider $A_{+}$. Using Green's formula, we obtain

$$
\int_{G} \phi(z)\left(d n_{\omega}(z)-d \mu(z)\right)=\frac{1}{2 \pi} \int_{G}(\Delta \phi)(z)\{\log |\psi(z, \omega)|-\log || \Psi(z)||\} d m(z) .
$$

Therefore,

$$
\begin{aligned}
\lambda \nu\left(A_{+}\right) & \leq \int_{A_{+}} d \nu(\omega) \int_{G} \phi(z)\left(d n_{\omega}(z)-d \mu(z)\right) \\
& =\frac{1}{2 \pi} \int_{G}(\Delta \phi)(z)\left\{\int_{A_{+}} \log |\psi(z, \omega)| d \nu(\omega)-\nu\left(A_{+}\right) \log \|\Psi(z)\|\right\} d m(z) .
\end{aligned}
$$


Then applying the lemma to the random variable $\mathcal{Z}(\omega)=\psi(., \omega)$ and to the set $A_{+}$(and recalling that $\|\Psi(z)\|$ is a variance of the gaussian random variable $\psi(z, \omega)$, cf. (4)), we proceed further:

$$
\begin{gathered}
\leq \frac{1}{2 \pi} \int_{G} \mid\left(\Delta \phi \left((z) \mid \nu\left(A_{+}\right)\left[\log \frac{1}{\nu\left(A_{+}\right)}+\frac{1}{4}\right] d m(z)\right.\right. \\
\quad=\frac{1}{2 \pi} \nu\left(A_{+}\right)\left[\log \frac{1}{\nu\left(A_{+}\right)}+\frac{1}{4}\right]\|\Delta \phi\|_{L^{1}} .
\end{gathered}
$$

That is,

or

$$
2 \pi \lambda \nu\left(A_{+}\right) \leq \nu\left(A_{+}\right)\left[\log \frac{1}{\nu\left(A_{+}\right)}+\frac{1}{4}\right]\|\Delta \phi\|_{L^{1}}
$$

$$
\nu\left(A_{+}\right) \leq e^{-2 \pi \lambda /\|\Delta \phi\|_{L^{1}}+1 / 4} .
$$

The same estimate holds for the set $A_{-}$, and this completes the proof.

Observe that the lemma also yields estimate (6) used in Section 2. For this, we apply the lemma to the same random variable $\mathcal{Z}(\omega)=\log |\psi(., \omega)|$ and to the sets

$$
A_{+}=\{\omega: \log |\mathcal{Z}(\omega)|-\log \|\Psi(z)\|>\lambda\}
$$

and

$$
A_{-}=\{\omega: \log |\mathcal{Z}(\omega)|-\log || \Psi(z) \|<-\lambda\} .
$$

We also observe, that applying the theorem with $\lambda=\int \phi d \mu$, we obtain

$$
\operatorname{Pr}\left(\left\{\omega: \int \phi d n_{\omega}=0\right\}\right) \leq 3 \exp \left[-2 \pi \int \phi d \mu /\|\Delta \phi\|_{1}\right] .
$$

This yields an upper bound for the "hole probability" $\operatorname{Pr}\left(\left\{\omega: n_{\omega}\left(D_{R}\right)=0\right\}\right)$. Let $D_{R} \subset G$ be a disk of radius $R$, and let $D_{r}, r<R$, be a concentric disk of a smaller radius $r$. Then we may choose a non-negative test-function $\phi(z)=\Phi(|z|)$ equal 1 on $D_{r}$, vanishing outside $D_{R}$, and such that

$$
\|\Delta \phi\|_{L^{1}}=\int_{r}^{R}\left(t\left|\Phi^{\prime \prime}(t)\right|+\left|\Phi^{\prime}(t)\right|\right) d t<C \frac{R+r}{R-r} .
$$

We obtain

\section{Corollary 1.}

$$
\operatorname{Pr}\left(\left\{\omega: n_{\omega}\left(D_{R}\right)=0\right\}\right) \leq 3 \exp \left[-c \mu\left(D_{r}\right) \frac{R-r}{R+r}\right], \quad 0<r<R .
$$

For example, if $\psi_{j}(z)$ are analytic in the unit disk $D$, and

$$
p=\operatorname{Pr}\left(\left\{\omega: n_{\omega}(D)=0\right\}\right)>0,
$$

then we get a dimensionless bound

$$
\mu\left(D_{r}\right) \leq C\left(\log \frac{3}{p}\right) \cdot \frac{1}{1-r}, \quad 0<r<1 .
$$


There is a certain resemblance between Offord's theorem and known results of the value-distribution theory due to Littlewood [19] and Ahlfors [1]. The reader may also have a look at Favorov's papers [8], [9], where infinite-dimensional versions of these results were obtained. We shall not pursue this matter here.

\section{Remarks}

1. Functions of several complex variables. The facts about zeros of gaussian analytic functions discussed above, after simple modifications, remain valid for gaussian analytic functions of several complex variables.

2. Holomorphic sections of line bundles. There is no need to assume that the analytic functions $\psi_{j}(z)$ are single-valued. Instead, one can deal, say, with zeros of character-automorphic functions or differential forms. More generally, one can work with zeros of gaussian holomorphic sections of a holomorphic line bundle. Since the proofs given above are "local", they carry over the case of holomorphic sections as well.

3. Non-gaussian distributions. In the proof of Theorem 1 , one can replace gaussian distributions by any rotational invariant distribution in $\mathbb{C}$ which does not charge the origin.

The Offord estimate is much more robust. The gaussian distribution was used only once during the estimate of concentration (18). For possible generalization of the lemma from Section 4 to more general classes of random variables see Offord's papers. A somewhat similar problem was treated by Favorov, Gorin and Ullrich, see [10], and references given there.

Added in proof:

Recently, A. Volberg and the author found another generalization of this lemma. Let $P$ be a polynomial in $\mathbb{R}^{n}$ of degree $d$, and let $d \nu$ be a logarithmically concave probability measure in $\mathbb{R}^{n}$ (that is, $d \nu=e^{-g(x)} d x$, where the set $K=\{g<+\infty\}$ and the function $\left.g\right|_{K}$ are convex). Then, for an arbitrary measurable set $E \subset \mathbb{R}^{n}$,

$$
\left|\frac{1}{\nu(E)} \int_{E} \log \right| P\left|d \nu-\int_{\mathbb{R}^{n}} \log \right| P|d \nu| \leq 2 d \log \frac{C}{\nu(E)} .
$$

This allows us to extend Theorem 3 to families of analytic functions which depend polynomially on real parameters.

\section{Acknowledgements}

Zeev Rudnick showed me a preprint version of the paper by Shiffman and Zelditch [26] and thus stimulated my interest in the subject. I thank Efim Gluskin, Leonid Pastur, Leonid Polterovich, Zeev Rudnick and Peter Yuditskii for useful discussions and comments. 


\section{References}

[1] L. Ahlfors, Ein Satz von Henri Cartan und seine Anwendung auf die Theorie der Meromorphen Funktionen, Soc. Sci. Fenn. Comment. Phys.-Math. 16 (1931), 1-19.

[2] P. Bleher, B. Shiffman, and S. Zelditch, Poincaré-Lelong approach to universality and scaling of correlations between zeros, preprint math-ph/9903012, 5 March, 1999.

[3] E. Bogomolny, O. Bohigas, and P. Leboeuf, Quantum chaotic dynamics and random polynomials, J. Statist.Phys. 85 (1996), 639-679.

[4] E. Calabi, Isometric imbedding of complex manifolds, Ann. of Math. 58 (1953), 1-23.

[5] _ Metric Riemann surfaces, Contribution to the theory of Riemann Surfaces, Annals of Mathematics Studies, no. 30., pp. 77-85 Princeton Univ. Press, Princeton, 1953.

[6] M.J. Cowen and R.G. Douglas, Complex geometry and operator theory, Acta Math. 141 (1978), 187-261.

[7] A. Edelman and E. Kostlan, How many zeros of a random polynomial are real? Bull. Amer. Math. Soc. 32 (1995), 1-37.

[8] S. Favorov, Growth and value distribution of maps from $\mathbb{C}^{m}$ into a Banach space, (Russian) Sibirsk. Mat. Zh. 31 (1990), 161-171; English translation in Siberian Math. J. 31 (1990), 137-146.

[9] Growth of holomorphic maps from finite dimensional space into a Banach space, (Russian) Mat. Fiz. Anal. Geom. 1 (1994), 240-251.

[10] _ A generalized Kahane-Khintchin inequality, Studia Math. 130 (1998), 101-107.

[11] P.J. Forrester and G. Honner, Exact statistical properties of the zeros of complex random polynomials, preprint cond-mat/9812388, 23 December, 1998.

[12] J.H. Hannay, Chaotic analytic zero points: exact statistics for those of a random spin state, J.Phys. A 29 (1996), L101-L105.

[13] _ The chaotic analytic function, J.Phys. A 31 (1998), L755-L761.

[14] J.P. Kahane, Some random series of functions, Second edition, Cambridge Studies in Advanced Mathematics, 5, Cambridge University Press, Cambridge-New York, 1985.

[15] _ Distribution des valuers des fonctions analytiques gaussiennes, Colloq. Math. 60 (1987), 175-187.

[16] H. Blaine Lawson Jr., The Riemannian geometry of holomorphic curves, Bull. Soc. Brasil Mat. 2 (1971), 45-62.

[17] P. Leboeuf, Random analytic chaotic eigenstates, preprint chao-dyn/9901019, 18 January, 1999.

[18] P. Leboeuf and P. Shukla, Universal fluctuations of zeros of chaotic wavefunctions, J. Phys. A 29 (1996), 4827-4835.

[19] J.E. Littlewood, Mathematical notes (11): On exceptional values of power series, J.London Math. Soc. 5 (1930), 82-87.

[20] N. Nikolskii, Distance formulae and invariant subspaces, with an application to localization of zeros of the Riemann S-function, Ann. Inst. Fourier (Grenoble) 45 (1995), 143-159.

[21] S. Nonnenmacher and A. Voros, Chaotic eigenfunctions in phase state, J. Statist. Phys. 92 (1998), 431-518.

[22] A.C. Offord, The distribution of zeros of power series whose coefficients are independent random variables, Indian J. Math. 9 (1967), 175-196.

[23] - The distribution of the values of a random function in the unit disk, Studia Math. 41 (1972), 71-106.

[24] (1972), 263-273.

[25] G. Pólya and G. Szegö, Problems and theorems in analysis, II, Theory of functions, zeros, polynomials, determinants, number theory, geometry, Springer Verlag, Berlin-New YorkHeidelberg, 1976. 
[26] B. Shiffman and S. Zelditch, Distribution of zeros of random and quantum chaotic sections of positive line bundles, Comm. Math. Phys. 200 (1999), 661-684.

School of Math. Sciences, Tel Aviv University, Ramat Aviv 69978, ISRAeL

E-mail address: sodin@math.tau.ac.il 